\title{
Are solar chromospheric fibrils tracing the magnetic field?
}

\author{
J. de la Cruz Rodríguez ${ }^{1,2}$ and H. Socas-Navarro ${ }^{3,4}$ \\ 1 Institute for Solar Physics, Royal Swedish Academy of Sciences, AlbaNova University Center, 10691 Stockholm, Sweden \\ e-mail: jaime@astro.su.se \\ 2 Department of Astronomy, Stockholm University, AlbaNova University Center, 10691 Stockholm, Sweden \\ 3 Instituto de Astrofísica de Canarias, Avda Vía Láctea S/N, La Laguna 38200, Tenerife, Spain \\ ${ }^{4}$ Departamento de Astrofísica, Universidad de La Laguna, 38205 La Laguna, Tenerife, Spain
}

Received 29 October 2010 / Accepted 17 January 2011

\section{ABSTRACT}

\begin{abstract}
Fibrils are thin elongated features visible in the solar chromosphere in and around magnetized regions. Because of their visual appearance, they have been traditionally considered a tracer of the magnetic field lines. For the first time, we challenge that notion by comparing their orientation to that of the magnetic field, obtained via high-resolution spectropolarimetric observations of Ca II lines. The short answer to the question posed in the title is that mostly yes, but not always.
\end{abstract}

Key words. Sun: activity - polarization - Sun: chromosphere - Sun: filaments, prominences - Sun: magnetic topology - sunspots

Narrow-band solar filtergrams in the H $6563 \AA(\mathrm{H} \alpha)$ line core display an ubiquitous pattern of fibrilar appearance covering most of the disk and often connecting patches of magnetic field. Although not as easily, the same pattern is also visible in the chromospheric Ca II lines (e.g. Zirin 1974; Marsh 1976; for a recent reference, see Pietarila et al. 2009). Fibril observation requires high spatial and spectral (particularly in $\mathrm{Ca}$ II) resolution, since they are very thin and observable only in the very core of the lines. Because of their visual appearance, which resembles magnetic field lines connecting the poles of a magnet, it has been traditionally assumed that fibrils indeed trace the chromospheric magnetic field. To the best of our knowledge, this common assumption has never been verified, probably because a proper empirical determination of the chromospheric magnetic field is very challenging, requiring high-resolution spectropolarimetry in chromospheric lines. Suitable instrumentation for this purpose has only just become available. Kuckein et al. (2010) were able to determine the vector field in filaments (where the magnetic field is relatively strong) using the TIP polarimeter (Collados et al. 1999).

The transverse (i.e., projected on the plane of the sky) component of the magnetic field, which is what we are interested in for this work, is determined solely by the observed linear polarization signals (Stokes $Q$ and $U$ profiles). Unfortunately, these signals are typically very weak and their observation presents numerous challenges. To extract a clear signal above the noise, we select by hand a small segment along the direction of a fibril and average the Stokes $Q$ and $U$ profiles spatially to improve the signal-to-noise ratio ( $Q$ and $U$ are averaged separately). From the profiles thus obtained, we can determine the azimuth of the magnetic field on the plane of the sky.

We present here results from two different datasets acquired with two different instruments, a Fabry-Perot interferometer and a slit spectro-polarimeter. Owing to the nature of the instrumentation employed, each dataset exhibits its own advantages and disadvantages for our purposes but they complement each other well, as we discuss below.
The first dataset is a spectro-polarimetric scan with the Spectro-Polarimeter for INfrared and Optical Regions (SPINOR, Socas-Navarro et al. 2006) of CaII $8542 \AA$ at the Dunn Solar Telescope of the National Solar Observatory/Sacramento Peak Observatory (Sunspot, NM, USA). The observing setup and the data are described in detail in that paper. We analyze a $(\lambda, x, y)$ cube acquired by scanning the spectrograph slit over a $80 \times 80^{\prime \prime}$ field of view. Seeing conditions were exceptionally good at the time and aided by the adaptive optics system (Rimmele 2000), we achieved a spatial resolution of approximately $0 . ' 6$ (although this figure varies during the scan because temporal fluctuations of the seeing). Because of its high quality, this dataset has also been used in previous papers studying the chromospheric field and electric currents in sunspots (Socas-Navarro 2005a,b). High spatial resolution is very important for the observation of fibrils, which are barely visible in typical spectroscopic observations of more modest resolution.

The second cube was acquired on 2008 June 6 with the Fabry-Perot interferometer CRisp Imaging Spectro-Polarimeter (CRISP, Scharmer 2006) in full Stokes mode at the Swedish 1-m Solar Telescope (SST, Scharmer et al. 2003). The Ca II $8542 \AA$ line was sampled at 17 wavelength points across the range $\pm 1.3 \AA$ from the core of the line, separated equidistantly by $162 \mathrm{~m} \AA$. The instrumental profile of CRISP has a full width half maximum of approximately $100 \mathrm{~m} \AA$ at $8542 \AA$. The images are processed using the image resconstruction code MoMFBD (van Noort et al. 2005), according to the scheme described in van Noort \& Rouppe van der Voort (2008) and Schnerr et al. (2011). The polarimetric response of the telescope is calibrated using a one meter polarizer, mounted on the entrance lens. Calibration images are used to fit the parameters of a theoretical model of the telescope as in Selbing (2005).

The SPINOR observations have higher spectral resolution than the CRISP ones $(120 \mathrm{~m} \AA$ dominated by instrumental resolution compared to $324 \mathrm{mÅ}$ dominated by spectral sampling, 


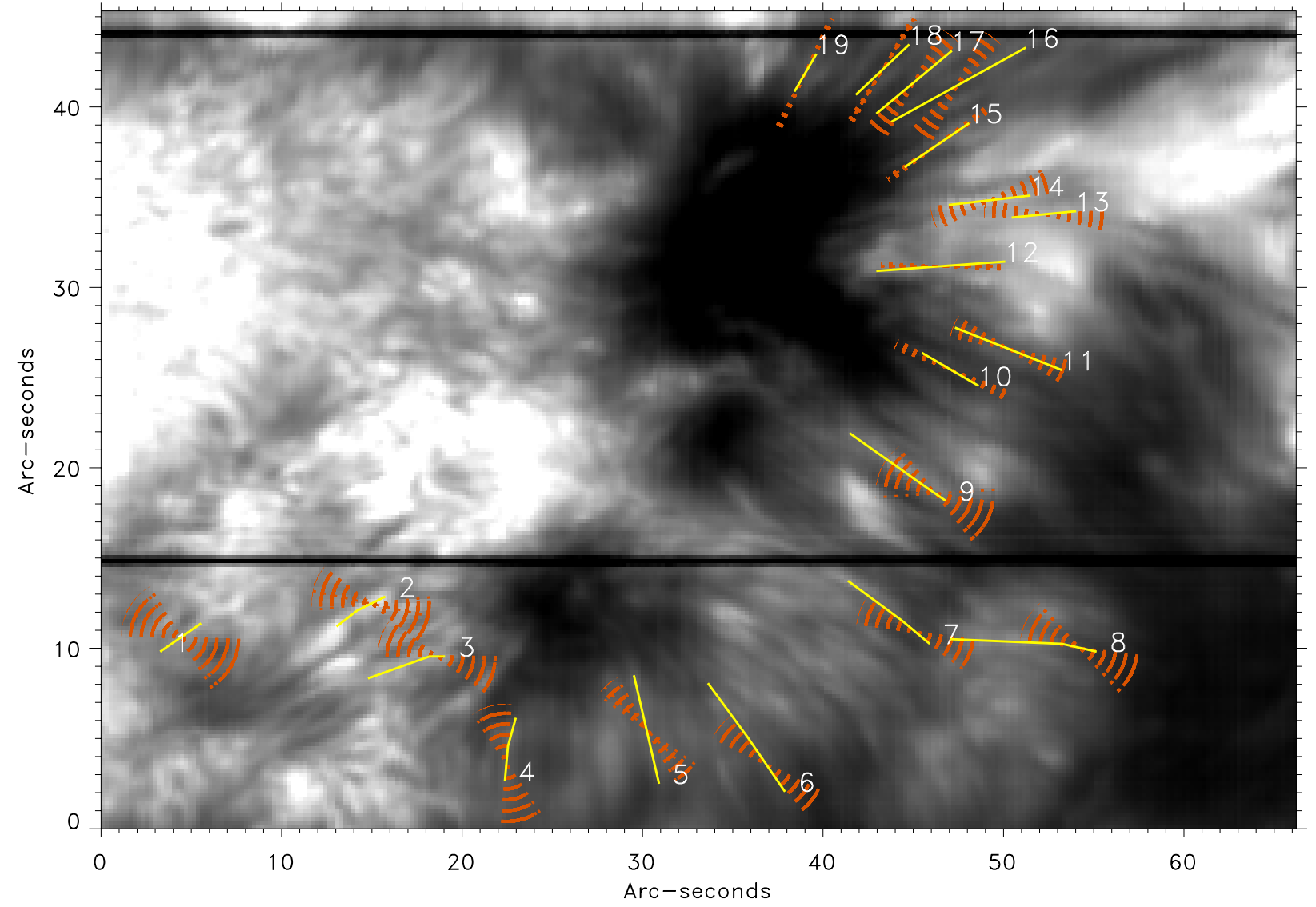

Fig. 1. Field of view observed with SPINOR in the core of Ca II $8542 \AA$. Yellow lines: fibrils selected for analysis. The yellow segments define the direction of the three-pixel wide bands used for Stokes $Q$ and $U$ profile averaging. Red cones: range of magnetic-field azimuth compatible with the $Q$ and $U$ profiles. The spatial sampling is 0 '.22/pixel.

respectively) and slightly higher polarimetric sensitivity. The noise in the Stokes parameters in the absence of signal (measured as the standard deviation in the continuum away from magnetic areas) is $4.5 \times 10^{-4}$ and $1.3 \times 10^{-3}$, respectively, in units of the average quiet-Sun continuum intensity. The spatial averaging of the profiles that we carry out in our analysis works well in improving the magnetic sensitivity as long as the observations are photon-noise limited. However, at some point one reaches a limit in which the uncertainties are dominated by other factors such as the goodness of the calibration, flat-fielding, spurious artifacts introduced in the image reconstruction process, and so forth. In the SPINOR case, this limit is reached at approximately $5 \times 10^{-5}$, whereas for CRISP it is around $10^{-4}$. The CRISP observations, on the other hand, have much higher spatial resolution $\left(0{ }^{\prime} .2\right.$ compared to $\left.0{ }^{\prime} 6\right)$ and the linear polarization reference direction is known. It is then possible to derive the absolute azimuth direction without any additional assumptions. For SPINOR, unfortunately, the linear polarization reference frame was not known and we need to resort to an a posteriori calibration of the zero azimuth using the data themselves. For this, we used a number of penumbral filaments visible in the sunspot photosphere and calculated the field azimuth in those locations as explained below. A constant offset was added to all the azimuth values and adjusted until the field orientation matched all the filaments simultaneously. The resulting offset uncertainty (which propagates directly into the fibril azimuth determinations) is approximately $2^{\circ}$.

In both cases (SPINOR and CRISP), we analyze observations of strong magnetic fields (in the vicinity of a sunspot) to ensure that the polarization induced in the spectral lines is produced by the Zeeman effect. In the quiet Sun, one would have to deal with complications due to the influence of the Hanle effect, which not only depolarizes the light (which would be irrelevant to our study) but also changes the relative amplitudes of Stokes $Q$ and $U$ (Manso Sainz \& Trujillo Bueno 2010). Most of the fibrils studied here are of a category that is sometimes referred to as superpenumbral fibrils (see e.g. Balasubramaniam et al. 2004) because they originate just beyond the boundary of a sunspot penumbra.

Figure 1 shows the chromospheric field of view observed with SPINOR in a grayscale image. Superimposed on that image, yellow lines indicate the fibril segments that we have manually selected for analysis based not only on the appearance of the fibrils but also on the presence of linear polarization. The Stokes profiles inside the band defined by each segment and a width of 3 pixels were averaged to produce one low-noise set of Stokes profiles for each fibril. The azimuth $\chi$ was then obtained using the following formula (Jefferies et al. 1989)

$$
\tan (2 \chi)=\frac{\int_{0}^{\infty} f(\lambda) U(\lambda)}{\int_{0}^{\infty} f(\lambda) Q(\lambda)}
$$

where $f(\lambda)$ is a bandwith selection function. In this case, we take $f(\lambda)$ to be a rectangular function of width $300 \mathrm{~m} \AA$ centered on the average position of the Stokes $Q$ and $U$ blue peak, very close to the line core. In the CRISP case, we take a single wavelength, where the observed linear polarization signal is maximal. The validity of this approximation is confirmed a posteriori since it 
Table 1. Fibril orientation $\chi_{\text {Fibril }}$ and magnetic-field azimuth $\chi_{\text {Field }}$ in the chromosphere of the SPINOR dataset.

\begin{tabular}{llll}
\hline \hline Index & $\chi_{\text {Fibril }}$ & $\chi_{\text {Field }} \pm \sigma_{\chi}$ & $|\Delta \chi| / \sigma_{\chi}$ \\
\hline 1 & 34.7 & $153.4 \pm 13.0$ & 9.13 \\
2 & 216.7 & $164.0 \pm 9.0$ & 5.86 \\
3 & 195.2 & $161.5 \pm 8.0$ & 4.22 \\
4 & 80.1 & $104.4 \pm 8.4$ & 2.91 \\
5 & 103.1 & $131.6 \pm 4.7$ & 6.06 \\
6 & 125.4 & $140.1 \pm 5.1$ & 2.91 \\
7 & 142.4 & $159.1 \pm 7.3$ & 2.28 \\
8 & 175.1 & $154.8 \pm 8.2$ & 2.49 \\
9 & 144.8 & $154.6 \pm 12.0$ & 0.81 \\
10 & 149.7 & $155.9 \pm 4.3$ & 1.43 \\
11 & 158.3 & $158.5 \pm 5.3$ & 0.03 \\
12 & 184.2 & $179.2 \pm 4.5$ & 1.11 \\
13 & 185.6 & $174.2 \pm 4.0$ & 2.83 \\
14 & 186.6 & $197.7 \pm 4.6$ & 2.42 \\
15 & 214.3 & $215.8 \pm 4.1$ & 0.35 \\
16 & 28.9 & $53.7 \pm 4.8$ & 5.18 \\
17 & 39.8 & $50.4 \pm 5.1$ & 2.10 \\
18 & 43.3 & $58.1 \pm 4.2$ & 3.54 \\
19 & 59.7 & $64.1 \pm 4.0$ & 1.08 \\
\hline
\end{tabular}

provides azimuth values that match the orientation of the photospheric penumbral filaments in the SPINOR dataset. We use this simpler procedure rather than full profile inversions because it works well in determining the azimuth and in this manner there is no need to deal with other complications inherent to the inversion process.

Our selection of fibrils is restricted to regions where polarization is detected after averaging. Unfortunately, the pixel-topixel profiles are too noisy to carry out individual measurements of the direction of the field. Only after averaging is the signal sufficiently high. For each fibril, the azimuth was calculated using Eq. (1). To estimate the error in the azimuth measurements, a Gaussian distribution of random values with the same sigma parameter as the standard deviation in the noise is added to $Q$ and $U$ and the azimuth is recomputed. This procedure is repeated 100 times with different realizations of the noise, yielding a total of 100 azimuth values. The spread in the results obtained allows us to estimate the uncertainties involved. The red cones drawn in the figure on the yellow segments outline all the azimuth realizations obtained for each fibril. Table 1 lists the values of azimuth obtained compared to the orientation of the fibrils. The rightmost column lists the discrepancy in units of the spread $\left(\sigma_{\chi}\right)$. When this value is significantly larger than three, we have a very high probability that the magnetic field orientation is incompatible with that of the fibril.

We note how most fibrils are aligned with the magnetic field, although there are a few noteworthy cases where significant misalignments occur, well above the observational error. The most obvious are located at the bottom left portion of the map (fibrils 1 through 5) near the smaller sunspot. Another interesting region is just above the large spot, where we find fibrils perfectly aligned with the field up until number 15 , then a large misalignment in number 16, which gradually decreases in 17 and 18 until finally number 19 is again well aligned.

For comparison, Fig. 2 shows the same for the photospheric penumbral filaments used to determine the absolute azimuth reference position. The $Q$ and $U$ signals are stronger inside the penumbra, which is why the uncertainties are smaller. The solar limb is towards the right in the figure and therefore the transverse component is stronger on the right-hand side of the penumbra

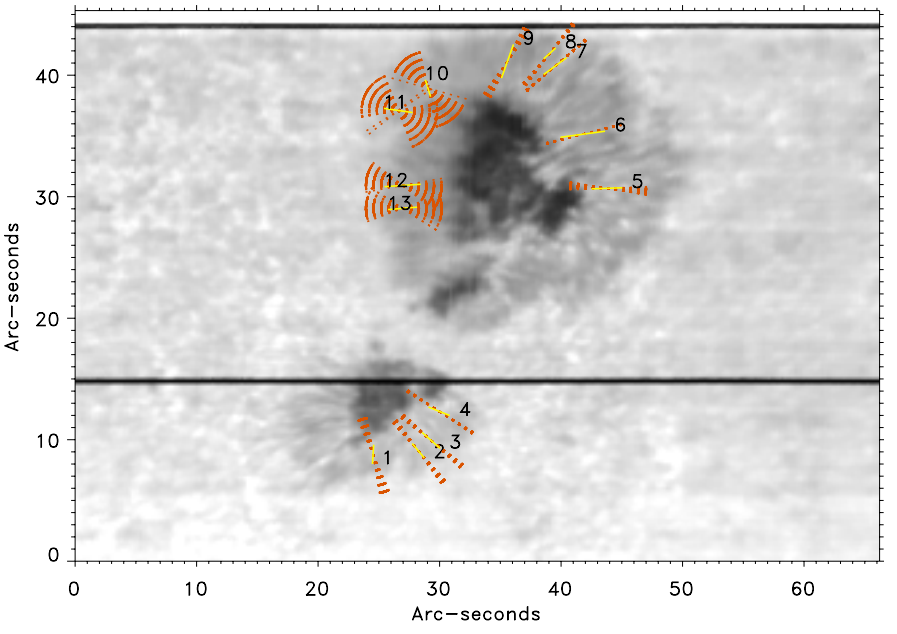

Fig. 2. Field of view observed with SPINOR in the wings of Ca II 8542 A. Yellow lines: penumbral filaments selected for analysis. The yellow segments define the direction of the three-pixel wide bands used for Stokes $Q$ and $U$ profile averaging. Red cones: range of magnetic field azimuth compatible with the $Q$ and $U$ profiles. The spatial sampling is $0.22 /$ pixel.

Table 2. Fibril orientation $\chi_{\text {Fibril }}$ and magnetic field azimuth $\chi_{\text {Field }}$ in the chromosphere of the CRISP dataset.

\begin{tabular}{llll}
\hline \hline Index & $\chi_{\text {Fibril }}$ & $\chi_{\text {Field }} \pm \sigma_{\chi}$ & $|\Delta \chi| / \sigma_{\chi}$ \\
\hline 1 & 176.1 & $175.6 \pm 11.5$ & 0.04 \\
2 & 185.7 & $165.8 \pm 4.4$ & 4.53 \\
3 & 178.0 & $171.1 \pm 4.2$ & 1.65 \\
4 & 180.0 & $150.2 \pm 3.5$ & 8.55 \\
5 & 196.9 & $157.9 \pm 8.0$ & 4.85 \\
6 & 226.1 & $137.3 \pm 3.0$ & 29.97 \\
7 & 28.9 & $109.6 \pm 6.5$ & 12.41 \\
8 & 48.3 & $65.7 \pm 14.6$ & 1.19 \\
9 & 62.3 & $106.2 \pm 6.1$ & 7.19 \\
10 & 67.9 & $55.2 \pm 5.9$ & 2.15 \\
11 & 92.1 & $93.0 \pm 10.1$ & 0.09 \\
12 & 82.6 & $85.4 \pm 4.6$ & 0.60 \\
13 & 126.3 & $75.4 \pm 9.4$ & 5.43 \\
\hline
\end{tabular}

due to projection effects. Notice how in this figure the field is much better aligned with the filaments.

We observe similar behavior in the CRISP data, for which the absolute azimuth reference is known (see Fig. 3 and Table 2). Most fibrils that show polarization signal have a magnetic field that is oriented along the fibril direction, at least within the margin allowed by the data. However, some areas (e.g., the region with fibrils number 4, 6, 7, and 9) have a field orientation that differs significantly from that of the fibrils.

In the light of these results, we conclude that the widespread idea that chromospheric fibrils are a visual proxy for the magnetic field lines may need to be reconsidered. Here we have limited ourselves to presenting observational evidence. An attempt to explain the appearance of the chromospheric fibrilar pattern and the nature of fibrils themselves is beyond the scope of the present study. We speculate that perhaps the small difference in formation height between the Ca II line core (where the fibrils are seen) and the Stokes $Q$ and $U$ peaks (where the magnetic field is measured) might explain the discrepant behavior. This would be a very surprising result because conceiving a field topology with such strong vertical gradients in field orientation, especially in the low- $\beta$ realm of the chromosphere (where 


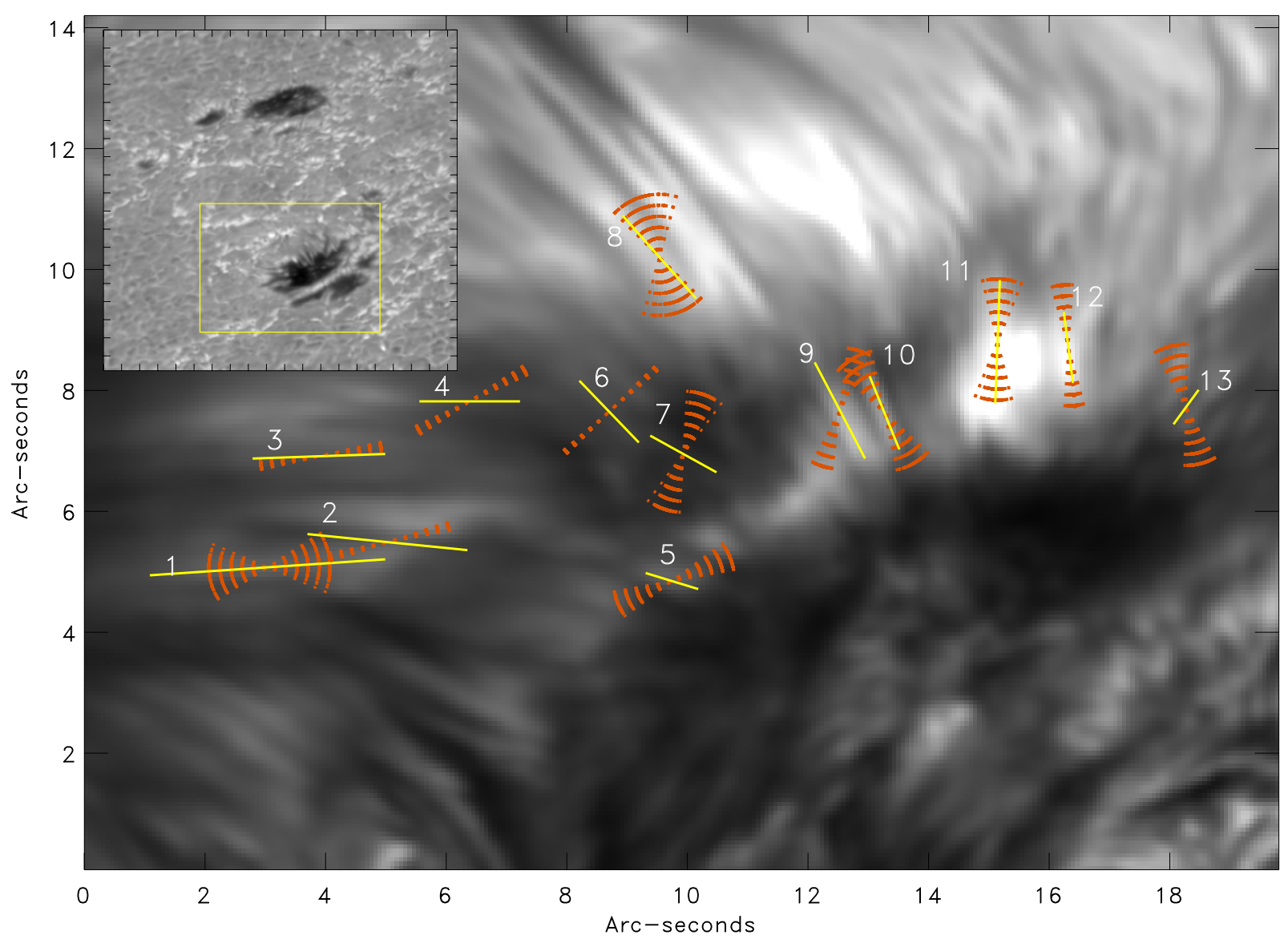

Fig. 3. Field of view observed with CRISP in the wing of Ca II $8542 \AA$. Inset: detailed view of the line core in the region of interest. Yellow lines: fibrils selected for analysis. The yellow segments define the direction of the three-pixel wide bands used for Stokes $Q$ and $U$ profile averaging. Red cones: range of magnetic-field azimuth compatible with the $Q$ and $U$ profiles. The intensity scale has been saturated to enhance the contrast of fibrils. The tick-mark separation of the inset is $2^{\prime \prime}$ covering an area of $39 \times 38^{\prime \prime}$ on the surface of the Sun.

magnetic field pressure and tensions are more difficult to sustain as the field dominates the dynamics of the plasma) is very challenging. Another possibility could be that the field changes rapidly in time and the plasma temperature structure (which is what ultimately dictates the intensity pattern observed) lags behind it somehow. Or perhaps the explanation is an entirely different one. In any case, we point out that the linear polarization signal observed in the chromosphere around sunspots weakens very abruptly as one moves outwards from the edge of the penumbra, a finding that is very difficult to reconcile with the large size of the fibrilar patterns that are seen around it, if these fibrils are indeed magnetic field lines, because in that case the chromospheric field strength (and the linear polarization signal) should not drop off so abruptly as it is observed.

Although the data and the analyses presented here are reasonably well established, our work obviously has some limitations. Given the relevance of this subject, additional observational studies should be conducted to confirm our refute these results. The main goal of our Letter is to draw attention to the subject since modern instrumentation now enables the determination of the magnetic field vector in fibrils. A more definite and comprehensive answer to the question raised in this paper should be something that can be realistically expected for the near future with the existing and upcoming tools for solar observations and their interpretation.

Acknowledgements. We are grateful to Luc Rouppe van der Voort for providing the SST/CRISP observations. This research project has been supported by a Marie Curie Early Stage Research Training Fellowship of the European
Communityâ Sixth Framework Programme under contract number MEST-CT2005-020395: The USO-SP International School for Solar Physics.

Financial support by the Spanish Ministry of Science and Innovation through project AYA2010-18029 (Solar Magnetism and Astrophysical Spectropolarimetry) is gratefully acknowledged by HSN.

\section{References}

Balasubramaniam, K. S., Pevtsov, A., \& Rogers, J. 2004, ApJ, 608, 1148 Collados, M., Rodríguez Hidalgo, I., Bellot Rubio, L., Ruiz Cobo, B., \& Soltau, D. 1999, in Astronomische Gesellschaft Meeting Abstracts, 13

Jefferies, J., Lites, B. W., \& Skumanich, A. 1989, ApJ, 343, 920

Kuckein, C., Centeno, R., \& Martínez Pillet, V. 2010, Mem. Soc. Astron. Ital., 81,668

Manso Sainz, R., \& Trujillo Bueno, J. 2010, ApJ, 722, 1416

Marsh, K. A. 1976, Sol. Phys., 50, 37

Pietarila, A., Hirzberger, J., Zakharov, V., \& Solanki, S. K. 2009, A\&A, 502, 647 Rimmele, T. R. 2000, in Adaptive Optical Systems Technology, ed. L. Peter, Wizinowich, Proc. SPIE, 4007, 218

Scharmer, G. B. 2006, A\&A, 447, 1111

Scharmer, G. B., Bjelksjo, K., Korhonen, T. K., Lindberg, B., \& Petterson, B. 2003, in Presented at the Society of Photo-Optical Instrumentation Engineers (SPIE) Conference, ed. S. L. Keil, \& S. V. Avakyan, 4853, 341

Schnerr, R. S., de la Cruz Rodríguez, J., \& van Noort, M. 2011, A\&A, submitted [arXiv: 1012.1225]

Selbing, J. 2005, Master's thesis, Stockholm University

Socas-Navarro, H. 2005a, ApJ, 633, L57

Socas-Navarro, H. 2005b, ApJ, 631, L167

Socas-Navarro, H., Elmore, D., Pietarila, A., et al. 2006, Sol. Phys., 235, 55 van Noort, M., Rouppe van der Voort, L., \& Löfdahl, M. G. 2005, Sol. Phys., 228,191

van Noort, M. J., \& Rouppe van der Voort, L. H. M. 2008, A\&A, 489, 429

Zirin, H. 1974, Sol. Phys., 38, 91 